TRANSACTIONS OF THE

AMERICAN MATHEMATICAL SOCIETY

Volume 356, Number 11, Pages 4601-4622

S 0002-9947(04)03537-8

Article electronically published on April 27, 2004

\title{
EXISTENCE AND CHARACTERIZATION OF REGIONS MINIMIZING PERIMETER UNDER A VOLUME CONSTRAINT INSIDE EUCLIDEAN CONES
}

\author{
MANUEL RITORÉ AND CÉSAR ROSALES
}

\begin{abstract}
We study the problem of existence of regions separating a given amount of volume with the least possible perimeter inside a Euclidean cone. Our main result shows that nonexistence for a given volume implies that the isoperimetric profile of the cone coincides with the one of the half-space. This allows us to give some criteria ensuring existence of isoperimetric regions: for instance, local convexity of the cone at some boundary point.

We also characterize which are the stable regions in a convex cone, i.e., second order minima of perimeter under a volume constraint. From this it follows that the isoperimetric regions in a convex cone are the euclidean balls centered at the vertex intersected with the cone.
\end{abstract}

\section{INTRODUCTION}

Let $M \subset \mathbb{R}^{n+1}$ be a solid cone, a cone over a connected, open subset $C$ with smooth boundary of the unit sphere $\mathbb{S}^{n}$. In this work we shall study if it is possible to separate a region $\Omega$ of given volume in $M$ with the least possible perimeter. This means that

$$
\mathcal{P}(\Omega, M) \leqslant \mathcal{P}(E, M)
$$

for all $E \subset M$ such that $\operatorname{vol}(E)=\operatorname{vol}(\Omega)$. Here $\mathcal{P}(\cdot, M)$ denotes the perimeter relative to $M$ and $\operatorname{vol}(\cdot)$ the $(n+1)$-dimensional Hausdorff measure in $\mathbb{R}^{n+1}$. If such an $\Omega$ exists we shall call it an isoperimetric region of volume $\operatorname{vol}(\Omega)$.

We remark that only the boundary area of $\Omega$ inside $M$ contributes to the perimeter, while $\partial \Omega \cap \partial M$ does not. Hence our problem is quite different from the one of enclosing a given volume with the least possible perimeter (the area of the hypersurface $\partial \Omega \cap \partial M$ is now taken into account), which has been recently studied by E. Stredulinsky and W. P. Ziemer $[\mathrm{StZ}$ and C. Rosales $\mathrm{R}$ inside a convex body. Since a cone $M$ contains round balls of any radius, the isoperimetric inequality in Euclidean space implies that round balls are the solutions to this second problem.

The problem of separating a given volume inside a convex body in Euclidean space has been studied by P. Sternberg and K. Zumbrun [SZ1, SZ2 and A. Ros and E. Vergasta [RV]. Existence for this problem is guaranteed by the compactness of the ambient manifold. In these papers some properties of the minimizing sets,

Received by the editors March 6, 2003 and, in revised form, July 22, 2003.

2000 Mathematics Subject Classification. Primary 53C20, 49Q20.

Key words and phrases. Isoperimetric regions, stability, hypersurfaces with constant mean curvature.

Both authors were supported by MCyT-Feder research project BFM2001-3489. 
such as connectedness of the boundary and estimates on the genus of the free boundary in the three-dimensional case, are obtained.

To decide whether isoperimetric regions exist or not in a noncompact manifold is not an easy problem. The only general results, to our knowledge, have been proved by F. Morgan for homogeneous manifolds (see the remark below the proof of Theorem 13.4 in [M3]) and by M. Ritoré [R2 for convex surfaces. There exist examples R1] of complete surfaces of revolution for which there are no isoperimetric regions of any given area. In [R2 it was shown that the direct method of the calculus of variations (take a minimizing sequence and extract a convergent subsequence) cannot be applied in the noncompact setting since part or all of a minimizing sequence could diverge.

We start this work by proving a general result, Theorem 2.1 on the behavior of minimizing sequences. They can be broken in two parts: passing to a subsequence, one converges to an isoperimetric region for the volume it encloses, and the other one diverges. In some cases it is easier to allow part of the sequence to diverge in order to get a contradiction, as was done in [R2] for complete convex surfaces. A survey of known regularity results completes Section 2 of the paper.

In Section 3 we treat the existence problem of isoperimetric regions in a cone $M \subset \mathbb{R}^{n+1}$. A simple observation, due to the presence of dilations, allows us to conclude that the isoperimetric dimension [Gr, $\S 6.4]$ of the cone is $(n+1)$, and that existence of isoperimetric regions for one given volume implies existence for all volumes. Since the cone is asymptotically flat, it is not difficult to show that the isoperimetric profile of the cone (see the notation subsection below for a precise definition) is bounded above by the isoperimetric profile of the half-space (Proposition 3.3). Then we prove in Theorem 3.4 by using Theorem 2.1 and a result by P. Bérard and D. Meyer $\mathrm{BM}$, that, in the case of nonexistence of isoperimetric regions for a given volume, the isoperimetric profile of $M$ is the one of the half-space. This is a strong result that gives us existence of isoperimetric regions under several conditions on the cone: for instance, when $M$ is a cone over a domain $C \subset \mathbb{S}^{n}$, with $\mathcal{H}^{n}(C) \leqslant \mathcal{H}^{n}\left(\mathbb{S}^{n}\right) / 2$ (Proposition 3.5), or when the boundary of the cone admits a local supporting hyperplane (Proposition 3.6). In particular, for a convex cone, there is existence for any value of volume. We also prove that any isoperimetric region in a cone is bounded in Proposition 3.7

In Section 4 we show which are the stable regions in a convex cone $M$. We define them as regions bounded by hypersurfaces of constant mean curvature $H$ with a singular set of small Hausdorff dimension such that the second derivative of perimeter is nonnegative for any volume preserving variation. In Theorem 4.9 we prove that the only stable regions in a convex cone are round balls contained in the closure of the cone, or balls centered at the vertex intersected with the cone, or half-balls centered and lying over a flat piece of $\partial M$. The method we use to prove Theorem 4.9 was introduced by J. L. Barbosa and M. do Carmo in [BdC] to show that round spheres are the only compact stable constant mean curvature hypersurfaces in $\mathbb{R}^{n+1}$, and was adapted later by F. Morgan and M. Ritoré $\mathrm{MR}$ to identify compact stable hypersurfaces of constant mean curvature in certain cones, allowing the presence of small singular sets. Once Theorem 4.9 is proved, a simple comparison allows us to characterize the isoperimetric regions in a convex cone in Theorem 4.11; they are the round balls centered at the vertex intersected with the cone. This result was first obtained by P. L. Lions and F. Pacella [LP] by using 
Brunn-Minkowski theory. In [M2 remark after Theorem 10.6], Frank Morgan has pointed that this result could also be obtained by modifying Gromov's proof of the isoperimetric inequality in Euclidean space.

We have also included in a final section as an appendix, a direct proof of Theorem 4.11 without using stability. The idea is as follows: for any isoperimetric region $\Omega$ we consider the set of equidistant hypersurfaces to its boundary; then, for a certain function depending on the relative profile of the equidistants it is satisfied that the second derivative with respect to the volume is nonnegative. From this, we deduce the result after an explicit calculation of the derivative above. The possible presence (in high dimensions) of singularities in the boundary of $\Omega$ requires approximation. Similar approximation arguments were used by F. Morgan and D. Johnson [MJ] to obtain a differential inequality for the isoperimetric profile in a compact Riemannian manifold.

Notation and preliminaries. Let $M^{n+1}$ be an open connected set of a smooth, complete, $(n+1)$-dimensional Riemannian manifold. The $(n+1)$-dimensional and the $k$-dimensional Hausdorff measures of a set $\Omega \subseteq M$ will be denoted by $\operatorname{vol}(\Omega)$ and $\mathcal{H}^{k}(\Omega)$, respectively. For any measurable set $\Omega \subseteq M$ and any open set $U \subseteq M$, let $\mathcal{P}(\Omega, U)$ be the perimeter of $\Omega$ relative to $U$, defined as

$$
\mathcal{P}(\Omega, U)=\sup \left\{\int_{\Omega} \operatorname{div} Y d \mathcal{H}^{n+1}:|Y| \leqslant 1\right\},
$$

where $Y$ is a smooth vector field with compact support contained in $U$, and $\operatorname{div} Y$ is the divergence of $Y$ Ch p. 3]. We shall simply denote $\mathcal{P}(\Omega)=\mathcal{P}(\Omega, M)$.

A set $\Omega$ is said to be of finite perimeter in $U$ if $\operatorname{vol}(\Omega \cap U)<\infty$ and $\mathcal{P}(\Omega, U)<\infty$. This condition is equivalent to requiring that the characteristic function of $\Omega$ is of bounded variation in $U$. In the case $U=M$ we simply say that $\Omega$ is of finite perimeter. For further background about perimeter and sets of finite perimeter we refer the reader to $\mathrm{Gi}$ and $[\mathrm{Z}]$.

As the perimeter of a set is not changed by adding or removing sets of $\mathcal{H}^{n+1}$. measure 0 , we shall always assume that $0<\operatorname{vol}\left(\Omega \cap B_{r}(p)\right)<\operatorname{vol}\left(B_{r}(p)\right)$, for every ball $B_{r}(p)$ centered at $p \in \partial \Omega$.

The isoperimetric profile of $M$ is the function $I_{M}:(0, \operatorname{vol} M) \rightarrow \mathbb{R}^{+} \cup\{0\}$ given by

$$
I_{M}(V)=\inf \{\mathcal{P}(\Omega): \Omega \subseteq M, \operatorname{vol}(\Omega)=V\} .
$$

An isoperimetric region in $M$ for volume $V \in(0, \operatorname{vol} M)$ is a set $\Omega \subseteq M$ satisfying $\operatorname{vol}(\Omega)=V$ and $\mathcal{P}(\Omega)=I_{M}(V)$. A minimizing sequence of sets of volume $V$ is a sequence of sets of finite perimeter $\left\{\Omega_{k}\right\}_{k \in \mathbb{N}}$ such that $\operatorname{vol}\left(\Omega_{k}\right)=V$ for all $k \in \mathbb{N}$ and $\lim _{k \rightarrow \infty} \mathcal{P}\left(\Omega_{k}\right)=I_{M}(V)$. We recall that a sequence $\left\{\Omega_{k}\right\}_{k \in \mathbb{N}}$ converges in the finite perimeter sense to a set $\Omega$ if $\left\{f_{k}\right\} \rightarrow f$ in $L_{\text {loc }}^{1}(M)$ and $\lim _{k \rightarrow \infty} \mathcal{P}\left(\Omega_{k}\right)=\mathcal{P}(\Omega)$. Here, $f_{k}$ and $f$ denote the characteristic function of $\Omega_{k}$ and $\Omega$, respectively.

Finally, we remark the following consequence of the coarea formula BZ, Theorem 13.4.2]: For any measurable set $\Omega \subset M$, we can compute $\operatorname{vol}(\Omega)$ as

$$
\operatorname{vol}(\Omega)=\int_{0}^{+\infty} \mathcal{H}^{n}\left(\Omega \cap S_{t}\right) d t
$$

where $S_{t}$ is the metric sphere in $M$ of radius $t$ centered at a point $p_{0} \in M$. 


\section{MINIMIZING SEQUENCES AND REGULARITY OF ISOPERIMETRIC REGIONS IN A RIEMANNIAN MANIFOLD}

In this section we study the behavior of a minimizing sequence for fixed volume. We shall work in open sets of complete Riemannian manifolds. The perimeter will be the one relative to the open set. The technique can be extended, with no extra effort, to other situations. This kind of argument was first used by F. Morgan; see Theorem 13.4 in [M3].

Theorem 2.1. Let $M^{n+1}$ be a connected unbounded open set of a complete Riemannian manifold. For any minimizing sequence $\left\{\Omega_{k}\right\}_{k \in \mathbb{N}}$ of sets of volume $V$, there exist a finite perimeter set $\Omega \subset M$ and sequences of sets of finite perimeter $\left\{\Omega_{k}^{c}\right\}_{k \in \mathbb{N}},\left\{\Omega_{k}^{d}\right\}_{k \in \mathbb{N}}$, such that

(i) $\operatorname{vol}(\Omega) \leqslant V, \quad \mathcal{P}(\Omega) \leqslant I_{M}(V)$.

(ii) $\operatorname{vol}\left(\Omega_{k}^{c}\right)+\operatorname{vol}\left(\Omega_{k}^{d}\right)=V, \quad \lim _{k \rightarrow \infty}\left[\mathcal{P}\left(\Omega_{k}^{c}\right)+\mathcal{P}\left(\Omega_{k}^{d}\right)\right]=I_{M}(V)$.

(iii) The sequence $\left\{\Omega_{k}^{d}\right\}_{k \in \mathbb{N}}$ diverges.

(iv) Passing to a subsequence, we have $\lim _{k \rightarrow \infty} \operatorname{vol}\left(\Omega_{k}^{c}\right)=\operatorname{vol}(\Omega)$ and $\lim _{k \rightarrow \infty} \mathcal{P}\left(\Omega_{k}^{c}\right)=\mathcal{P}(\Omega)$. In fact, $\left\{\Omega_{k}^{c}\right\}_{k \in \mathbb{N}}$ converges to $\Omega$ in the finite perimeter sense.

(v) $\Omega$ is an isoperimetric region (it could be empty) for the volume it encloses.

Remark 2.2. For $n=1$ it was shown in [R2, Lemma 2.2] that the sets $\Omega_{k}^{c}$ and $\Omega_{k}^{d}$ can be taken as a union of connected components of $\Omega_{k}$.

Proof. Fix a point $p_{0} \in M$ and denote by $B_{r}$ (resp. $S_{r}$ ) the intersection of $M$ with the open ball (resp. sphere) in the ambient manifold centered at $p_{0}$ of radius $r>0$. Clearly $B_{m} \subset B_{m+1}$ for $m \in \mathbb{N}$ and $M=\bigcup_{m \in \mathbb{N}} B_{m}$.

Let $f_{k}$ be the characteristic function of $\Omega_{k}$. Applying the Compactness Theorem [Gi Theorem 1.19] on each $B_{m}$ and a diagonal argument, we get the existence of a function $f \in L_{\text {loc }}^{1}(M)$ and of a subsequence, that we denote again by $\left\{\Omega_{k}\right\}$, such that $\left\{f_{k}\right\} \rightarrow f$ in $L_{\text {loc }}^{1}(M)$. We can even assume that $\left\{f_{k}\right\}$ pointwise converges $\mathcal{H}^{n+1}$-almost everywhere to $f$, and so $f$ is the characteristic function of a set $\Omega \subset M$. By Fatou's Lemma and the lower semicontinuity of perimeter Gi. Theorem 1.9], we have $\operatorname{vol}(\Omega) \leqslant V$ and $\mathcal{P}(\Omega) \leqslant I_{M}(V)$. If $\operatorname{vol}(\Omega)=V$, then $\Omega$ is an isoperimetric region of volume $V$ and the conclusion follows by taking $\Omega_{k}^{c}=\Omega_{k}$ and $\Omega_{k}^{d}=\emptyset$ for all $k \in \mathbb{N}$. Henceforth we assume that $\operatorname{vol}(\Omega)<V$.

To define $\Omega_{k}^{c}$ and $\Omega_{k}^{d}$ we need to choose an appropriate truncation of $\Omega_{k}$. By the coarea formula, on any real interval $J$ we have $\int_{J} \mathcal{H}^{n}\left(\Omega_{k} \cap S_{t}\right) d t \leqslant \operatorname{vol}\left(\Omega_{k}\right)=V$. If length $(J) \geqslant \ell$, then there exists $t \in J$ such that

$$
\mathcal{H}^{n}\left(\Omega_{k} \cap S_{t}\right) \leqslant \frac{V}{\ell}
$$

From this observation, passing again to a subsequence of $\Omega_{k}$ if necessary, it is easy to find a sequence $r(k)$ of positive real numbers such that

$$
\begin{aligned}
r(k+1)-r(k) & \geqslant k, \\
\mathcal{H}^{n}\left(\Omega_{k} \cap S_{r(k+1)}\right) & \leqslant \frac{V}{k}, \\
\int_{B_{r(k+1)}}\left|f_{k}-f\right| d \mathcal{H}^{n+1} & \leqslant \frac{1}{k} .
\end{aligned}
$$


For each $k \in \mathbb{N}$, define $\Omega_{k}^{c}=\Omega_{k} \cap B_{r(k+1)}$ and $\Omega_{k}^{d}=\Omega_{k}-B_{r(k+1)}$. Clearly $\Omega_{k}=\Omega_{k}^{c} \cup \Omega_{k}^{d}$ and so $\operatorname{vol}\left(\Omega_{k}^{c}\right)+\operatorname{vol}\left(\Omega_{k}^{d}\right)=V$. Moreover, we can choose the sequence $\{r(k)\}_{k \in \mathbb{N}}$ so that [Z, Corollary 5.5.3]

$$
\begin{aligned}
& \mathcal{P}\left(\Omega_{k}^{c}\right) \leqslant \mathcal{P}\left(\Omega_{k}, B_{r(k+1)}\right)+\mathcal{H}^{n}\left(\Omega_{k} \cap S_{r(k+1)}\right) \\
& \mathcal{P}\left(\Omega_{k}^{d}\right) \leqslant \mathcal{P}\left(\Omega_{k}, M-\bar{B}_{r(k+1)}\right)+\mathcal{H}^{n}\left(\Omega_{k} \cap S_{r(k+1)}\right) .
\end{aligned}
$$

As $\operatorname{vol}\left(\Omega_{k}\right)=V$, we get from 2.2

$I_{M}(V) \leqslant \mathcal{P}\left(\Omega_{k}\right) \leqslant \mathcal{P}\left(\Omega_{k}^{c}\right)+\mathcal{P}\left(\Omega_{k}^{d}\right) \leqslant \mathcal{P}\left(\Omega_{k}\right)+2 \mathcal{H}^{n}\left(\Omega_{k} \cap S_{r(k+1)}\right) \leqslant \mathcal{P}\left(\Omega_{k}\right)+\frac{2 V}{k}$,

which implies that $\lim _{k \rightarrow \infty}\left[\mathcal{P}\left(\Omega_{k}^{c}\right)+\mathcal{P}\left(\Omega_{k}^{d}\right)\right]=I_{M}(V)$ and proves (ii). Assertion (iii) follows from the inclusion $\Omega_{k}^{d} \subset M-B_{r(k+1)}$.

To prove (iv) observe that

$$
\begin{aligned}
\left|\operatorname{vol}\left(\Omega_{k}^{c}\right)-\operatorname{vol}(\Omega)\right| & \leqslant\left|\operatorname{vol}\left(\Omega_{k} \cap B_{r(k+1)}\right)-\operatorname{vol}\left(\Omega \cap B_{r(k+1)}\right)\right|+\operatorname{vol}\left(\Omega-B_{r(k+1)}\right) \\
& \leqslant \int_{B_{r(k+1)}}\left|f_{k}-f\right| d \mathcal{H}^{n+1}+\operatorname{vol}\left(\Omega-B_{r(k+1)}\right),
\end{aligned}
$$

and so $\lim _{k \rightarrow \infty} \operatorname{vol}\left(\Omega_{k}^{c}\right)=\operatorname{vol}(\Omega)$ by $(2.3)$. On the other hand, by the definition of the sets $\Omega_{k}^{c}$ we have

$$
\int_{B_{r(k+1)}}\left|f_{t}^{c}-f\right| d \mathcal{H}^{n+1}=\int_{B_{r(k+1)}}\left|f_{t}-f\right| d \mathcal{H}^{n+1}, \quad t \geqslant k,
$$

where $f_{t}^{c}$ denotes the characteristic function of $\Omega_{t}^{c}$. The equality above gives us $\left\{f_{k}^{c}\right\}_{k} \rightarrow f$ in $L_{\text {loc }}^{1}(M)$. Hence $\mathcal{P}(\Omega) \leqslant \liminf _{k \rightarrow \infty} \mathcal{P}\left(\Omega_{k}^{c}\right)$ by the lower semicontinuity of perimeter.

To finish the proof we only have to see that $\Omega$ is perimeter minimizing among sets of volume $V_{0}=\operatorname{vol}(\Omega)$, and that $\liminf _{k \rightarrow \infty} \mathcal{P}\left(\Omega_{k}^{c}\right)=\mathcal{P}(\Omega)$. If any of these statements is not true, then we will construct an "improved minimizing sequence" to get a contradiction.

Suppose first that $\Omega$ is not an isoperimetric region. In this case, we can find a set $E \subset M$ of volume $V_{0}$ with smooth boundary (relative to $M$ ) such that $\mathcal{P}(E)<$ $\mathcal{P}(\Omega)$. From (2.1) there exists $t(k) \in(r(k), r(k+1))$ such that

$$
\mathcal{H}^{n}\left(E \cap S_{t(k)}\right) \leqslant \frac{V_{0}}{k}
$$

Let $E_{t(k)}=E \cap B_{t(k)}$. Obviously $\lim _{k \rightarrow \infty} \operatorname{vol}\left(E_{t(k)}\right)=V_{0}$.

Now, take a point $q_{0} \in \partial E \cap M$ and a small neighborhood $W$ of $q_{0}$ in $\partial E$. Let $u \not \equiv 0$ be a nonnegative function in $C_{0}^{\infty}(W)$. For all $k$ large enough $W \subset E_{t(k)}$ and $u$ induces a variation of $\partial E_{t(k)}$ that can be used to slightly modify the volume of $E_{t(k)}$. Since $\operatorname{vol}\left(E_{t(k)}\right) \rightarrow V_{0}$ and $\operatorname{vol}\left(\Omega_{k}^{d}\right) \rightarrow V-V_{0}$, we use the variation associated to $u$ to get, for large $k$, a set $E_{t(k)}^{\prime}$ so that

$$
\operatorname{vol}\left(E_{t(k)}^{\prime}\right)+\operatorname{vol}\left(\Omega_{k}^{d}\right)=V .
$$

By the first variation for perimeter and volume, we have

$$
\mathcal{P}\left(E_{t(k)}^{\prime}\right) \leqslant \mathcal{P}\left(E_{t(k)}\right)+\alpha\left|v_{k}\right|,
$$

where $v_{k}=\operatorname{vol}\left(E_{t(k)}^{\prime}\right)-\operatorname{vol}\left(E_{t(k)}\right)$ converges to 0 and $\alpha$ is a positive constant independent of $k$. 
Let $T_{k}=E_{t(k)}^{\prime} \cup \Omega_{k}^{d}$. The volume of $T_{k}$ equals $V$ and, by (2.4),

$$
\begin{aligned}
\mathcal{P}\left(T_{k}\right) & \leqslant \mathcal{P}\left(E, B_{t(k)}\right)+\mathcal{H}^{n}\left(E \cap S_{t(k)}\right)+\alpha\left|v_{k}\right|+\mathcal{P}\left(\Omega_{k}^{d}\right) \\
& \leqslant \mathcal{P}(E)+\frac{V_{0}}{k}+\alpha\left|v_{k}\right|+\mathcal{P}\left(\Omega_{k}^{d}\right),
\end{aligned}
$$

from which we obtain

$$
\liminf _{k \rightarrow \infty} \mathcal{P}\left(T_{k}\right) \leqslant \mathcal{P}(E)+\liminf _{k \rightarrow \infty} \mathcal{P}\left(\Omega_{k}^{d}\right)<\mathcal{P}(\Omega)+\liminf _{k \rightarrow \infty} \mathcal{P}\left(\Omega_{k}^{d}\right) \leqslant I_{M}(V),
$$

since $\mathcal{P}(\Omega) \leqslant \liminf _{k \rightarrow \infty} \mathcal{P}\left(\Omega_{k}^{c}\right)$. By this contradiction (v) is proved. To finish the proof of (iv) we proceed in a similar way; in fact we only have to work with $\Omega$ instead of $E$. We must remark that this can be done since the set of regular points in $\partial \Omega \cap M$ is open; see Proposition 2.4 below.

Remark 2.3. From Theorem 2.1 we obtain the existence of minimizers of any given volume when $M$ is unbounded and of finite volume.

We finish this section by recalling smoothness properties of the boundary of an isoperimetric region in an open subset $M^{n+1}$ of an $(n+1)$-dimensional Riemannian manifold. Regularity results for isoperimetric hypersurfaces in the Euclidean setting were obtained by E. Gonzalez, U. Massari and I. Tamanini [GMT, who treated interior regularity, and by M. Grüter [G1], who studied regularity near boundary points. As pointed out by F. Morgan [M3 their results can also be stated in the setting of Riemannian manifolds, by using the paper of F. Almgren $\underline{\text { A }}$. A complete proof of interior regularity for isoperimetric boundaries in Riemannian manifolds can be found in [M1] weaker regularity properties when $\partial M$ is not $C^{\infty}$, but only $C^{1,1}$, are also established. We collect the results above in the next proposition.

Proposition 2.4 (Regularity). Let $\Omega$ be a perimeter-minimizing set under a volume constraint in a connected open set $M^{n+1}$ with smooth boundary $\partial M$ of an $(n+1)$ dimensional Riemannian manifold. Then, the boundary $\Lambda=\overline{\partial \Omega \cap M}$ can be written as a disjoint union $\Sigma \cup \Sigma_{0}$, where $\Sigma$ is the regular part of $\Lambda$ and $\Sigma_{0}=\Lambda-\Sigma$ is the set of singularities. Moreover, we have

(i) $\Sigma \cap M$ is a smooth, embedded hypersurface with constant mean curvature with respect to the inner normal.

(ii) If $x \in \Sigma \cap \partial M$, then $\Sigma$ is a smooth, embedded hypersurface with boundary contained in $\partial M$ in a neighborhood of $x$; in this neighborhood $\Sigma$ has constant mean curvature and meets $\partial M$ orthogonally.

(iii) $\Sigma_{0}$ is a closed set of Hausdorff dimension less than or equal to $n-7$.

Remark 2.5. In the preceding proposition the regular set $\Sigma$ is defined as follows: for $x \in \Sigma$ there is a neighborhood $W$ of $x$ in $\Sigma$ such that $W$ is a smooth, embedded hypersurface without boundary or with boundary contained in $\partial M$. Note that one of the conclusions of the proposition above is the absence of interior points in $\Sigma$ meeting $\partial M$ tangentially; see [G2].

Example. This example illustrates that isoperimetric regions in a smooth manifold with boundary need not meet the boundary of the manifold.

Let $M$ be the compact surface obtained from attaching the hemisphere of $\mathbb{S}^{2}$ centered at the north pole to the compact cylinder $\mathbb{S}^{1} \times[-h, 0](h>\pi)$ through the circle $\mathbb{S}^{1} \times\{0\}$. Suppose that $\Omega$ is an isoperimetric region in $M$ of area $A>2 \pi$ such that $\Lambda=\overline{\partial \Omega \cap M}$ meets $\partial M$ orthogonally. As the cylinder is locally isometric 
to the half-plane at any point of $\partial M$, we deduce that $\Lambda$ contains either a semicircle centered at $\partial M$ or two vertical segments $\left\{\theta_{i}\right\} \times[-h, 0], i=1,2$. In any of these cases we have $\mathcal{P}(\Omega)^{2}>4 \pi^{2}=\mathcal{P}(D)^{2}$, where $D$ is the geodesic disk in $M$ centered at the north pole and enclosing area $A$. This contradiction shows that any isoperimetric region $\Omega$ of area $A>2 \pi$ satisfies $\Lambda \cap \partial M=\emptyset$. If $\bar{\Omega} \cap \partial M$ is nonvoid, then $\Omega^{\prime}=M-\Omega$ is an isoperimetric region that does not meet $\partial M$.

\section{Existence of isoperimetric REgions inside a EuClidean CONE}

In this section we shall consider a cone $M=0 \circledast C$, where $C$ is a connected open set (domain) in $\mathbb{S}^{n} \subset \mathbb{R}^{n+1}$ with smooth boundary. The closure of the cone is $\bar{M}=0 \times \bar{C}$. When $C$ is an open half-sphere the cone coincides with an open halfspace. It is well known that the isoperimetric regions in a half-space are half-balls centered at the boundary of the half-space [SZ1, and that the isoperimetric profile of the half-space is given by

$$
I_{n+1}(V)=\left(\frac{\mathbf{c}_{n}}{2}\right)^{1 /(n+1)}(n+1)^{n /(n+1)} V^{n /(n+1)}, \quad V>0,
$$

where $\mathbf{c}_{n}=\mathcal{H}^{n}\left(\mathbb{S}^{n}\right)$.

We begin with two easy consequences of the invariance of a cone by dilations.

Proposition 3.1. The isoperimetric profile $I_{M}$ of a cone $M$ satisfies

$$
I_{M}(V)=c V^{n /(n+1)} \quad \text { for } V>0,
$$

where $c$ is a constant equal to $I_{M}\left(V_{0}\right) / V_{0}^{n /(n+1)}$ for any $V_{0}>0$. Hence $I_{M}^{(n+1) / n}(V)$ is a linear function of $V$.

Proof. For any $\lambda>0$ let $h_{\lambda}(p)=\lambda p$. Fix $V_{0}>0$ and consider a finite perimeter set $\Omega \subset M$ with $\operatorname{vol}(\Omega)=V_{0}$. For each $\lambda>0$, the set $\Omega_{\lambda}=h_{\lambda}(\Omega)$ is contained in $M$ and satisfies $\operatorname{vol}\left(\Omega_{\lambda}\right)=\lambda^{n+1} V_{0}$ and $\mathcal{P}\left(\Omega_{\lambda}\right)=\lambda^{n} \mathcal{P}(\Omega)$. By the definition of $I_{M}$ we have $I_{M}\left(\lambda^{n+1} V_{0}\right) \leqslant \lambda^{n} \mathcal{P}(\Omega)$, and so $I_{M}\left(\lambda^{n+1} V_{0}\right) \leqslant \lambda^{n} I_{M}\left(V_{0}\right)$. With a similar argument we obtain the opposite inequality and

$$
I_{M}\left(\lambda^{n+1} V_{0}\right)=\lambda^{n} I_{M}\left(V_{0}\right) .
$$

From this equality the result follows by taking $\lambda=\left(V / V_{0}\right)^{1 /(n+1)}$.

Proposition 3.2. Let $M$ be a cone over a smooth domain in a sphere $\mathbb{S}^{n} \subset \mathbb{R}^{n+1}$. Then either there exist isoperimetric regions for any given value of volume, or there are no isoperimetric regions at all.

Proof. Let $h_{\lambda}(p)=\lambda p$. It is clear that $\Omega \subset M$ is an isoperimetric region of volume $V_{0}$ if and only if $h_{\lambda}(\Omega) \subset M$ is an isoperimetric region of volume $\lambda^{n+1} V_{0}$.

The following result gives an upper bound on the isoperimetric profile of a cone $M$ and it is a consequence of the fact that $\partial M$ is asymptotically flat.

Proposition 3.3. The isoperimetric profile $I_{M}$ of a cone $M \subset \mathbb{R}^{n+1}$ is bounded from above by the isoperimetric profile of the half-space.

Proof. Consider a diverging sequence of metric balls $D_{k}^{\prime}$ centered at points of $\partial M$, so that the volume of $D_{k}=D_{k}^{\prime} \cap M$ equals $V$. By the definition of isoperimetric profile we have $I_{M}(V) \leqslant \mathcal{P}\left(D_{k}\right)$ for all $k \in \mathbb{N}$. On the other hand $\lim _{k \rightarrow \infty} \mathcal{P}\left(D_{k}\right)=$ $I_{n+1}(V)$ since $\partial M$ approaches a hyperplane at infinity. Thus,

$$
I_{M}(V) \leqslant \lim _{k \rightarrow \infty} \mathcal{P}\left(D_{k}\right)=I_{n+1}(V) .
$$


The main result of this section is the following theorem.

Theorem 3.4. Let $M$ be a cone in $\mathbb{R}^{n+1}$. If there are not isoperimetric regions in $M$, then the isoperimetric profile $I_{M}$ of $M$ is the one of the half-space.

Proof. We know from Proposition 3.3 that inequality $I_{M}(V) \leqslant I_{n+1}(V)$ holds for all $V>0$. To see the reverse inequality suppose that there are not isoperimetric regions in $M$. From Theorem 2.1 this means that the volume of the sets of the convergent part of any minimizing sequence of sets of volume $V$ goes to zero. Hence there exists a diverging sequence $\left\{\Omega_{k}\right\}_{k \in \mathbb{N}}$ of sets of finite perimeter in $M$ such that

(i) $\operatorname{vol}\left(\Omega_{k}\right) \leqslant V$ for all $k \in \mathbb{N}$.

(ii) $\lim _{k \rightarrow \infty} \operatorname{vol}\left(\Omega_{k}\right)=V$.

(iii) $\lim _{k \rightarrow \infty} \mathcal{P}\left(\Omega_{k}\right)=I_{M}(V)$.

Fix an arbitrary $\varepsilon>0$. By the Bérard-Meyer isoperimetric inequality BM] App. C], there exists a constant $V_{\varepsilon}>0$ such that, for any set of finite perimeter $\Omega \subset B_{4}-\bar{B}_{1}$ with $\operatorname{vol}(\Omega) \leqslant V_{\varepsilon}$, we get

$$
\mathcal{P}\left(\Omega, B_{4}-\bar{B}_{1}\right)^{(n+1) / n} \geqslant c_{n}(1-\varepsilon)^{(n+1) / n} \operatorname{vol}(\Omega),
$$

where $B_{r}$ is the intersection of the open ball of radius $r$ centered at the origin with the cone, and $c_{n}$ is the constant that appears in the expression of the isoperimetric profile of the half-space $I_{n+1}(V)^{(n+1) / n}=c_{n} V$. By scaling we get that, for any finite perimeter set $\Omega \subset B_{4 \lambda}-\bar{B}_{\lambda}$ such that $\operatorname{vol}(\Omega) \leqslant \lambda^{n+1} V_{\varepsilon}$, we have

$$
\mathcal{P}\left(\Omega, B_{4 \lambda}-\bar{B}_{\lambda}\right)^{(n+1) / n} \geqslant c_{n}(1-\varepsilon)^{(n+1) / n} \operatorname{vol}(\Omega) .
$$

Let $S_{r}$ be the intersection of the sphere of radius $r>0$ centered at the vertex of the cone with the cone.

Since the sequence $\left\{\Omega_{k}\right\}_{k}$ diverges, we may assume, passing to a subsequence if necessary, that $\bar{\Omega}_{m} \subset M-\bar{B}_{m}$, for all $m \in \mathbb{N}$.

Fix $m \in \mathbb{N}$ so that $m^{n+1} \geqslant V / V_{\varepsilon}$. For such $m$ we use the coarea formula to define a sequence $\{t(q)\}_{q \in \mathbb{N}}$ of positive real numbers by setting $t(0)=m$ and $t(q) \in\left(2^{q-1} m, 2^{q} m\right), q \geqslant 1$, so that

$$
\mathcal{H}^{n}\left(\Omega_{m} \cap S_{t(q)}\right) \leqslant \frac{\operatorname{vol}\left(\Omega_{m}\right)}{2^{q-1} m} \leqslant \frac{V}{2^{q-1} m}, \quad q \geqslant 1 .
$$

Obviously $t(1) / t(0) \leqslant 2$ and $t(q) / t(q-1)<4$ for $q \geqslant 2$.

Define $\Omega_{m}^{q}=\Omega_{m} \cap\left(B_{t(q)}-\bar{B}_{t(q-1)}\right)$. The set $\Omega_{m}^{q}$ is contained in $B_{t(q)}-\bar{B}_{t(q-1)}$. We have $t(q)<4 t(q-1)$, and also

$$
\operatorname{vol}\left(\Omega_{m}^{q}\right) \leqslant \operatorname{vol}\left(\Omega_{m}\right) \leqslant V \leqslant m^{n+1} V_{\varepsilon} \leqslant t(q-1)^{n+1} V_{\varepsilon} .
$$

From inequality (3.1) we get

$$
\mathcal{P}\left(\Omega_{m}^{q}, B_{4 t(q-1)}-\bar{B}_{t(q-1)}\right)^{(n+1) / n} \geqslant c_{n}(1-\varepsilon)^{(n+1) / n} \operatorname{vol}\left(\Omega_{m}^{q}\right), \quad q \in \mathbb{N},
$$

and so, from inequality $\sum_{i} a_{i}^{(n+1) / n} \leqslant\left(\sum_{i} a_{i}\right)^{(n+1) / n}$,

$$
\left[\sum_{q=1}^{\infty} \mathcal{P}\left(\Omega_{m}^{q}, B_{4 t(q-1)}-\bar{B}_{t(q-1)}\right)\right]^{(n+1) / n} \geqslant c_{n}(1-\varepsilon)^{(n+1) / n} \operatorname{vol}\left(\Omega_{m}\right) .
$$

Taking into account that

$\mathcal{P}\left(\Omega_{m}^{q}, B_{4 t(q-1)}-\bar{B}_{t(q-1)}\right) \leqslant \mathcal{P}\left(\Omega_{m}^{q}, B_{t(q)}-\bar{B}_{t(q-1)}\right)+\mathcal{H}^{n}\left(\Omega_{m} \cap S_{t(q)}\right), \quad q \in \mathbb{N}$, 
and that

$$
\sum_{q=1}^{\infty} \mathcal{P}\left(\Omega_{m}^{q}, B_{t(q)}-\bar{B}_{t(q-1)}\right)=\sum_{q=1}^{\infty} \mathcal{P}\left(\Omega_{m}, B_{t(q)}-\bar{B}_{t(q-1)}\right) \leqslant \mathcal{P}\left(\Omega_{m}\right)
$$

we have, from (3.2),

$$
\mathcal{P}\left(\Omega_{m}\right)+\sum_{q=1}^{\infty} \frac{V}{2^{q-1} m} \geqslant \sum_{q=1}^{\infty} \mathcal{P}\left(\Omega_{m}^{q}, B_{4 t(q-1)}-\bar{B}_{t(q-1)}\right) .
$$

From this inequality and (3.3), letting $m \rightarrow \infty$ we conclude that

$$
I_{M}(V)^{(n+1) / n} \geqslant c_{n}(1-\varepsilon)^{(n+1) / n} V=(1-\varepsilon)^{(n+1) / n} I_{n+1}(V)^{(n+1) / n} .
$$

As $\varepsilon$ is arbitrary the proof follows.

Now, we establish some criteria to decide whether or not there exist isoperimetric regions in a cone.

Proposition 3.5. Let $M \subset \mathbb{R}^{n+1}$ be a cone over a domain $C \subset \mathbb{S}^{n}$ such that $\mathcal{H}^{n}(C) \leqslant \mathbf{c}_{n} / 2$, where $\mathbf{c}_{n}$ is the Riemannian volume of $\mathbb{S}^{n}$. Then isoperimetric regions exist in $M$ for any value of volume.

In particular, isoperimetric regions of any volume exist in a convex cone.

Proof. Simply observe that the perimeter and the volume of a ball $B_{r}$ of radius $r>0$ centered at the origin intersected with $M$ are given by

$$
\mathcal{P}\left(B_{r}\right)=r^{n} \mathcal{H}^{n}(C), \quad \operatorname{vol}\left(B_{r}\right)=\frac{r^{n+1}}{n+1} \mathcal{H}^{n}(C) .
$$

The relative isoperimetric profile of these balls equals

$$
\mathcal{P}(V)=\mathcal{H}^{n}(C)^{1 /(n+1)}(n+1)^{n /(n+1)} V^{n /(n+1)}
$$

which is less than or equal to the isoperimetric profile of the half-space. Hence the first part of the proposition follows by Theorem 3.4.

If $M$ is convex, then $\mathcal{H}^{n}(C) \leqslant \mathbf{c}_{n} / 2$ and we apply the first part of the proposition.

Another criterion for existence of isoperimetric regions in a cone is given in the next proposition.

Proposition 3.6. Consider a cone $M$ over a smooth domain $C \subset \mathbb{S}^{n}$, and assume that $\partial M-\{0\}$ has a point $x$ admitting a local supporting hyperplane. Then isoperimetric regions exist in $M$ for any value of volume.

Proof. Let $\mathcal{P}(r)$ and $V(r)$ be the perimeter and the volume of the ball $B_{r}$ of radius $r>0$ centered at $x$ intersected with the cone, and $\widetilde{V}(r)$ the volume of the cone subtended by $\partial B_{r} \cap M$ and vertex at $x$. We have the relation

$$
\mathcal{P}(r)=(n+1) \frac{\tilde{V}(r)}{r} .
$$

Since $M$ is locally convex near $x, V(r) \geqslant \widetilde{V}(r)$ for $r$ small, so that

$$
\mathcal{P}(r)=(n+1) \frac{\tilde{V}(r)}{r} \leqslant(n+1) \frac{V(r)}{r} .
$$


On the other hand, if $\mathcal{P}_{e}(r)$ and $V_{e}(r)$ are the area and volume of the half-ball of radius $r>0$, we have

$$
\frac{\mathcal{P}_{e}(r)}{V_{e}(r)}=\frac{n+1}{r}
$$

and so

$$
\frac{\mathcal{P}(r)}{V(r)} \leqslant \frac{\mathcal{P}_{e}(r)}{V_{e}(r)} .
$$

Since $V(r) \leqslant V_{e}(r)$ for $r$ small, by the local convexity of $\partial M$ around $x$, we get

$$
\frac{\mathcal{P}(r)}{V(r)^{n /(n+1)}}=\frac{\mathcal{P}(r)}{V(r)} V(r)^{1 /(n+1)} \leqslant \frac{\mathcal{P}_{e}(r)}{V_{e}(r)} V_{e}(r)^{1 /(n+1)}=\frac{\mathcal{P}_{e}(r)}{V_{e}(r)^{n /(n+1)}}=d_{n},
$$

where $d_{n}$ is the constant that appears in the expression of the isoperimetric profile of the half-space $I_{n+1}(V)=d_{n} V^{n /(n+1)}$.

Hence, for small $r, \mathcal{P}(r) \leqslant I_{n+1}(V(r))$. By Theorem 3.4 isoperimetric regions exist in the cone for any value of volume.

The last result of this section shows the boundedness of an isoperimetric region in a cone. The proof is modelled on the Euclidean one [M3 Lemma 13.6]. We include it here for the sake of completeness.

Proposition 3.7. Any isoperimetric region $\Omega$ in a cone $M$ is bounded.

Proof. For any $r \geqslant 0$ denote $V(r)=\operatorname{vol}\left(\Omega-B_{r}\right)$ and $\mathcal{P}(r)=\mathcal{P}\left(\Omega, M-\bar{B}_{r}\right)$, where $B_{r}$ is the open ball of radius $r$ centered at the origin. The function $V(r)$ is decreasing and satisfies $\lim _{r \rightarrow \infty} V(r)=0$. By the coarea formula we have $V^{\prime}(r)=$ $-\mathcal{H}^{n}\left(\Omega \cap \partial B_{r}\right)$ for almost all $r \geqslant 0$.

By applying the isoperimetric inequality of the cone in Proposition 3.1 and [Z] Corollary 5.5.3] we obtain

$$
\left|V^{\prime}(r)\right|+\mathcal{P}(r) \geqslant \mathcal{P}\left(\Omega-B_{r}\right) \geqslant c V(r)^{n /(n+1)},
$$

for almost all $r \geqslant 0$.

Now, take a large $r \geqslant 0$ so that $V(r)$ is close enough to zero. By (i) in Proposition 2.4 we can select an open subset $W$ of regular points of $\partial \Omega \cap M$ contained in $\Omega \cap B_{r}$, and a nonnegative smooth function $u$ with compact support contained in $W$. This function corresponds to a normal variation of $W$ and, by the first variation formulae for perimeter and volume, $d \mathcal{P} / d V\left(\operatorname{vol}\left(\Omega \cap B_{r}\right)\right)=n H(H$ is the constant mean curvature of $W$ ). Hence, if we produce a small increment of volume $V(r)$, then the increment of perimeter is less than or equal to $(1+n H) V(r)$. By using that $\Omega$ is an isoperimetric region we deduce that

$$
\mathcal{P}\left(\Omega, B_{r}\right)+\mathcal{H}^{n}\left(\Omega \cap \partial B_{r}\right)+(1+n H) V(r) \geqslant \mathcal{P}(\Omega) \geqslant \mathcal{P}\left(\Omega, B_{r}\right)+\mathcal{P}(r),
$$

which implies that

$$
\left|V^{\prime}(r)\right|+(1+n H) V(r) \geqslant \mathcal{P}(r), \quad \text { for almost all } r \geqslant 0 .
$$

Adding inequalities (3.4) and (3.5) and using the fact that $\lim _{r \rightarrow+\infty} V(r)=0$, we obtain

$$
2\left|V^{\prime}(r)\right| \geqslant \frac{c}{2} V(r)^{n /(n+1)}, \quad \text { for almost all } r \gg 0 .
$$

Now, if we admit that $\Omega$ is unbounded, then $V(r)>0$ and

$$
(n+1)\left(V^{1 /(n+1)}\right)^{\prime}(r)=-\left|V^{\prime}(r)\right| V(r)^{-n /(n+1)} \leqslant-\frac{c}{4},
$$

which is clearly a contradiction since $V(r)$ is positive for all $r>0$. 


\section{Characterization of stable Regions WITH SMALL SINGULAR SET IN CONVEX CONES}

We begin this section with a general notion of stability for sets of finite perimeter. Let us consider a cone $M \subset \mathbb{R}^{n+1}$ and a bounded set of finite perimeter $\Omega \subset$ $M$. We take a vector field $X$ in $\mathbb{R}^{n+1}$ with compact support such that $X(p) \in$ $T_{p}(\partial M)$ for all $p \in \partial M-\{0\}$ and we consider the associated one-parameter group of diffeomorphisms $\left\{\varphi_{t}\right\}_{t}$ of $\bar{M}$.

We shall say that $\Omega_{t}=\varphi_{t}(\Omega)$ is a volume-preserving deformation of $\Omega$ if $\operatorname{vol}\left(\Omega_{t}\right)=$ $\operatorname{vol}(\Omega)$ for $t$ small enough. Let $\mathcal{P}(t)=\mathcal{P}\left(\Omega_{t}\right)$. It is said that $\Omega$ is stationary if $\mathcal{P}^{\prime}(0)=0$ for all volume-preserving deformations. It is said that $\Omega$ is stable if it is stationary and $\mathcal{P}^{\prime \prime}(0) \geqslant 0$ for all volume-preserving deformations. Obviously any isoperimetric region in $M$ is a stable region. Theorems 4.9 and 4.11 show that, in general, there are stable regions inside a cone which are not isoperimetric.

From now on we assume that $\Lambda=\overline{\partial \Omega \cap M}$ can be decomposed as $\Sigma \cup \Sigma_{0}$, where $\Sigma_{0}$ is a closed singular set consisting of isolated points or such that $\mathcal{H}^{n-2}\left(\Sigma_{0}\right)=0$, and $\Sigma$ is a smooth, embedded hypersurface such that $\partial \Sigma-\Sigma_{0} \subset \partial M$. We assume that $0 \in \Sigma_{0}$ in the case $0 \in \Lambda$. It must be remarked that we are not excluding the possibility that $\Sigma$ and $\partial M$ are tangent at some interior point to $\Sigma$. We define $\partial^{*} \Sigma=\partial \Sigma-\Sigma_{0}$. If $\partial^{*} \Sigma=\emptyset$, we adopt the convention that the integrals over this set are all equal to 0 .

The setting above allows us to give an analytical expression for stationarity and stability from the variational formulae for perimeter and volume. If $X$ is a vector field over $M$ with compact support on the regular boundary $\Sigma$ and such that $X(p) \in T_{p}(\partial M)$ whenever $p \in \partial M-\{0\}$, then $X$ induces a variation of $\Omega$ for which the derivatives of perimeter and volume are given by

$$
\mathcal{P}^{\prime}(0)=-\int_{\Sigma} n H u d \mathcal{H}^{n}-\int_{\partial^{*} \Sigma}\langle X, \nu\rangle d \mathcal{H}^{n-1}, \quad V^{\prime}(0)=-\int_{\Sigma} u d \mathcal{H}^{n},
$$

where $H$ is the mean curvature with respect to a unit normal vector field $N, u=$ $\langle X, N\rangle$ is the normal component of the variation, and $\nu$ is the inward normal vector to $\partial^{*} \Sigma$ in $\Sigma$. Hence, if $\Omega$ is stationary, then $H$ is constant and $\Sigma$ meets $\partial M$ orthogonally in the points of $\partial^{*} \Sigma$, SZ1.

The derivative $\mathcal{P}^{\prime \prime}(0)$ of perimeter when $\Omega$ is stationary and the variation preserves volume is given by $[\mathrm{RV}]$

$$
Q(u, u)=-\int_{\Sigma} u\left(\Delta u+|\sigma|^{2} u\right) d \mathcal{H}^{n}-\int_{\partial^{*} \Sigma} u\left\{\frac{\partial u}{\partial \nu}+\operatorname{II}(N, N) u\right\} d \mathcal{H}^{n-1}
$$

where $\Delta$ is the Laplacian on $\Sigma,|\sigma|^{2}$ is the squared sum of the principal curvatures associated to $N$, and II is the second fundamental form of $\partial M-\{0\}$ with respect to the inner normal. The equation above defines a quadratic form on $C_{0}^{\infty}(\Sigma)$ called the index form.

As in [BdC], a function $u \in C_{0}^{\infty}(\Sigma)$ satisfying $\int_{\Sigma} u d \mathcal{H}^{n}=0$ induces a volumepreserving deformation of $\Omega$ with associated vector field $X$ so that the normal component $\langle X, N\rangle$ equals $u$. Thus, if $\Omega$ is stable, then we have $Q(u, u) \geqslant 0$ for any function $u$ with compact support and mean zero on $\Sigma$. We conclude that if $\Omega$ is a stable region in $M$, then $\Sigma$ is a stable constant mean curvature hypersurface as defined in $\mathrm{BdC}$ and $\mathrm{RV}$. Moreover, for a function $u \in C_{0}^{\infty}(\Sigma)$, integration by 
parts gives another expression for the index form, namely

$$
I(u, u)=\int_{\Sigma}\left(|\nabla u|^{2}-|\sigma|^{2} u^{2}\right) d \mathcal{H}^{n}-\int_{\partial^{*} \Sigma} \operatorname{II}(N, N) u^{2} d \mathcal{H}^{n-1},
$$

where $\nabla u$ is the gradient of $u$ on $\Sigma$. The right side of (4.2) has meaning for functions in the Sobolev space $H^{1}(\Sigma)$ with compact support in $\Sigma$.

Inequality $Q(u, u) \geqslant 0$ gives interesting geometrical and topological information when a suitable function $u$ is inserted. Following [BdC] we shall prove Theorem 4.9 by considering the test function $u=1+H\langle X, N\rangle$, where $X$ is the position vector field in $\mathbb{R}^{n+1}$ given by $X(p)=p$. It was shown by $\mathrm{H}$. Wente $[\mathrm{W}$ ] that the function $u$ appears when one considers first a contraction of a smooth hypersurface by parallels and then applies a dilation to restore the enclosed volume.

In our problem, we cannot insert the function $u$ in the index form (4.1) since its support is not included in $\Sigma$. Our first objective is extending the validity of inequality $Q(u, u) \geqslant 0$ to more general functions. We need some preliminary results.

Remark 4.1. If $\Omega$ is a bounded set of finite perimeter in a cone $M$, then $\Omega$ has finite perimeter in $\mathbb{R}^{n+1}$ by [Gi, Theorem 2.10], and so the Divergence Theorem is valid in $\Omega$ for vector fields $X \in C_{0}^{\infty}\left(\mathbb{R}^{n+1}, \mathbb{R}^{n+1}\right)$. In particular, if $X(p) \in T_{p}(\partial M)$ for any $p \in \partial M-\{0\}$, then

$$
\int_{\Omega} \operatorname{div} X d \mathcal{H}^{n+1}=-\int_{\partial \Omega \cap M}\langle X, N\rangle d \mathcal{H}^{n},
$$

where $N$ is the generalized inner normal to $\Omega$; see [Z, Theorem 5.8.2].

Lemma 4.2. Let $\Omega$ be a bounded set of finite perimeter in $M$ with $\Lambda=\Sigma \cup \Sigma_{0}$, where $\Sigma_{0}$ is a closed singular set such that $\mathcal{H}^{n-2}\left(\Sigma_{0}\right)=0$ or consists of isolated points, and $\Sigma$ is a smooth, embedded hypersurface with $\partial \Sigma-\Sigma_{0} \subset \partial M$.

If $\Omega$ is stationary, then there exists a positive constant $\mu$ (not depending on $r$ ) such that

$$
\frac{\mathcal{H}^{n}\left(\Sigma \cap B_{r}\right)}{r^{n}} \leqslant \mu
$$

for almost all open balls $B_{r} \subset \mathbb{R}^{n+1}$ with small enough radius.

Proof. Let $H$ be the constant mean curvature of $\Sigma$ with respect to the normal $N$ which points into $\Omega$. We follow the proofs of the monotonicity formulae established in [S] and [GJ], so we need to show that $\Sigma$ has generalized mean curvature in the sense of [S, 17.1] and [GJ, p. 133, (7)]. That is, we need to establish

$$
\int_{\Sigma} \operatorname{div}_{\Sigma} X d \mathcal{H}^{n}=-n H \int_{\Sigma}\langle X, N\rangle d \mathcal{H}^{n}
$$

for vector fields $X$ with support in a small ball (here $\operatorname{div}_{\Sigma} X$ denotes the divergence on $\Sigma$ ). Following $[\mathrm{S}$ ] and [G.J], the formula above specialize to appropriate vector fields and the boundedness of the mean curvature of $\Sigma$ give the monotonicity formulae for interior balls [S. Theorem 17.6], and for balls meeting $\partial M-\{0\}$; see [GJ] Theorem 3.4]. On the other hand, the vector field $X(p)=p$ is tangent to $\partial M$ and so the proof of L. Simon shows that the monotonicity formula for interior balls also holds for small balls centered at the vertex of the cone. From these formulae the proof of the lemma follows.

Let us prove (4.4). Consider a small open ball $B$ contained in the interior of the cone or centered at a point of $\Lambda \cap \partial M$, and a vector field $X \in C_{0}^{\infty}\left(B, \mathbb{R}^{n+1}\right)$ which 
vanishes at the origin and such that $X(p) \in T_{p}(\partial M)$ for all $p \in \partial M-\{0\}$. Let $\left\{\varphi_{t}\right\}_{t}$ of $X$ be the one-parameter group of diffeomorphisms associated to $X$.

Now, take a point $q_{0} \in(\Sigma \cap M)-\bar{B}$ and a small Euclidean ball $B^{\prime}$ disjoint from $B$, and let $W=B^{\prime} \cap \Sigma$. Let $u \in C_{0}^{\infty}(W)$ such that $-\int_{W} u d \mathcal{H}^{n}=1$. It is possible to construct a vector field $Y$ in $B^{\prime}$ with $\left.Y\right|_{W}=u N$ with associated flow $\left\{\psi_{s}\right\}_{s}$.

Consider the two-parameter variation $\psi_{s}\left(\varphi_{t}(\Omega)\right)$, and let

$$
\operatorname{vol}(s, t)=\operatorname{vol}\left(\psi_{s}\left(\varphi_{t}(\Omega)\right)\right)
$$

and $\mathcal{P}(s, t)=\mathcal{P}\left(\psi_{s}\left(\varphi_{t}(\Omega)\right)\right)$. By the first variation for volume and (4.3) we have

$$
\operatorname{vol}_{t}(0,0)=\int_{\Omega} \operatorname{div} X d \mathcal{H}^{n+1}=-\int_{\Sigma}\langle X, N\rangle d \mathcal{H}^{n}, \quad \operatorname{vol}_{s}(0,0)=-\int_{\Sigma} u d \mathcal{H}^{n}=1
$$

By the Implicit Function Theorem, there is a function $s(t)$, with $s(0)=0$ and $s^{\prime}(0)=\int_{\Sigma}\langle X, N\rangle d \mathcal{H}^{n}$, such that $\operatorname{vol}(s(t), t)=\operatorname{vol}(\Omega)$.

On the other hand, the first variation formula of perimeter for varifolds $[S, 16.2]$ and the fact that $\operatorname{div}_{\Sigma} Y=-n H u$ imply

$$
\mathcal{P}_{t}(0,0)=\int_{\Sigma} \operatorname{div}_{\Sigma} X d \mathcal{H}^{n}, \quad \mathcal{P}_{s}(0,0)=\int_{\Sigma} \operatorname{div}_{\Sigma} Y d \mathcal{H}^{n}=n H .
$$

Since $\Omega$ is stationary and the variation $\psi_{s(t)}\left(\varphi_{t}(\Omega)\right)$ preserves the volume of $\Omega$ we get that the derivative of perimeter with respect to this variation vanishes. A direct computation using that the supports of $X$ and $Y$ are disjoint shows that this derivative equals

$$
\begin{aligned}
0 & =\mathcal{P}_{t}(0,0)+s^{\prime}(0) \mathcal{P}_{s}(0,0) \\
& =\int_{\Sigma} \operatorname{div}_{\Sigma} X d \mathcal{H}^{n}+n H \int_{\Sigma}\langle X, N\rangle d \mathcal{H}^{n}
\end{aligned}
$$

which proves (4.4).

The next lemma gives an approximation of the function 1 on $\Sigma$ by functions with compact support on $\Sigma$ with controlled gradient.

Lemma 4.3. Let $\Omega$ be a bounded set of finite perimeter in $M$ with $\Lambda=\Sigma \cup \Sigma_{0}$, where $\Sigma_{0}$ is a closed singular set such that $\mathcal{H}^{n-2}\left(\Sigma_{0}\right)=0$ or consists of isolated points, $\Sigma$ is a smooth, embedded hypersurface with boundary, and $\partial^{*} \Sigma=\partial \Sigma-\Sigma_{0}$ is contained in $\partial M$.

If $\Omega$ is stationary, then, given $\varepsilon>0$, there is a smooth function $\varphi_{\varepsilon}: \Sigma \rightarrow[0,1]$ with compact support, and such that

(i) $\mathcal{H}^{n}\left(\left\{\varphi_{\varepsilon} \neq 1\right\}\right) \leqslant \varepsilon, \quad \mathcal{H}^{n-1}\left(\left\{\left.\varphi_{\varepsilon}\right|_{\partial^{*} \Sigma} \neq 1\right\}\right) \leqslant \varepsilon$.

(ii) $\int_{\Sigma}\left|\nabla \varphi_{\varepsilon}\right|^{2} d \mathcal{H}^{n} \leqslant \varepsilon$.

Proof. Suppose that the singular set $\Sigma_{0}$ is nonvoid (otherwise it suffices to define $\varphi_{\varepsilon} \equiv 1$ for all $\varepsilon>0$ ). Assume first that $n \geqslant 3$; in this case it is clear that $\mathcal{H}^{n-2}\left(\Sigma_{0}\right)=0$ and we proceed exactly as in [SZ2, Lemma 2.4] to define the function $\varphi_{\varepsilon}$. On the other hand, for $n=2$ the set $\Sigma_{0}$ consists in a finite set of isolated boundary points and the construction of $\varphi_{\varepsilon}$ can be obtained as in MR, Lemma 3.1]. Both constructions use Lemma 4.2

The following result illustrates how to use Lemma4.3 in order to extend classical results to more general situations. Recall that if $\partial^{*} \Sigma=\emptyset$, then the corresponding integral is assumed to be zero. 
Lemma 4.4. Let $\Omega$ be a bounded set of finite perimeter in $M$ with $\Lambda=\Sigma \cup \Sigma_{0}$, where $\Sigma_{0}$ is a closed singular set such that $\mathcal{H}^{n-2}\left(\Sigma_{0}\right)=0$ or consists of isolated points, $\Sigma$ is a smooth, embedded hypersurface with boundary, and $\partial^{*} \Sigma=\partial \Sigma-\Sigma_{0}$ is contained in $\partial M$. If $\Omega$ is stationary, then

(i) (Divergence Theorem) If $X$ is a smooth, tangent vector field on $\Sigma$ satisfying $|X|^{2}, \operatorname{div} X \in L^{1}(\Sigma)$ and $\langle X, \nu\rangle \in L^{1}\left(\partial^{*} \Sigma\right)$, then

$$
\int_{\Sigma} \operatorname{div} X d \mathcal{H}^{n}=-\int_{\partial^{*} \Sigma}\langle X, \nu\rangle d \mathcal{H}^{n-1}
$$

where $\operatorname{div} X$ denotes the divergence relative to $\Sigma$, and $\nu$ is the inward normal vector to $\partial^{*} \Sigma$ in $\Sigma$.

(ii) (Integration by parts) For any smooth bounded function $u: \Sigma \rightarrow \mathbb{R}$ such that $|\nabla u|^{2},|\Delta u| \in L^{1}(\Sigma)$ and $\frac{\partial u}{\partial \nu} \in L^{1}\left(\partial^{*} \Sigma\right)$, it is satisfied that

$$
\int_{\Sigma}\left(u \Delta u+|\nabla u|^{2}\right) d \mathcal{H}^{n}=-\int_{\partial^{*} \Sigma} u \frac{\partial u}{\partial \nu} d \mathcal{H}^{n-1} .
$$

Proof. Let us prove (i). Consider a sequence of functions $\left\{\varphi_{\varepsilon}\right\}_{\varepsilon>0}$ as obtained in Lemma 4.3. Since the field $\varphi_{\varepsilon} X$ has compact support in $\Sigma$, we can apply the classical divergence theorem to obtain

$$
\int_{\Sigma} \operatorname{div}\left(\varphi_{\varepsilon} X\right) d \mathcal{H}^{n}=-\int_{\partial^{*} \Sigma} \varphi_{\varepsilon}\langle X, \nu\rangle d \mathcal{H}^{n-1}
$$

On the other hand, we have $\operatorname{div}\left(\varphi_{\varepsilon} X\right)=\varphi_{\varepsilon} \operatorname{div} X+\left\langle\nabla \varphi_{\varepsilon}, X\right\rangle$. Letting $\varepsilon \rightarrow 0$ in (4.5) and using the Cauchy-Schwarz inequality in $L^{2}(\Sigma)$, the properties of $\varphi_{\varepsilon}$, and the hypotheses on $X$, we prove (i). Statement (ii) follows from (i) by taking $X=u(\nabla u)$.

Example ([Wh $]$ ). This example illustrates that the Divergence Theorem for noncompact manifolds with boundary need not hold if we do not impose $|X|^{2} \in L^{1}(\Sigma)$. Let $\Sigma$ be the square $[0,1] \times[0,1]$, where the vertices have been suppressed. The vector field $X(p)=p /|p|^{2}$ satisfies

$$
0=\int_{\Sigma} \operatorname{div} X d \mathcal{H}^{2} \neq \int_{\partial \Sigma}\langle X, \nu\rangle d \mathcal{H}^{1}=\frac{\pi}{2}
$$

Now, we can extend the class of test functions for which inequality $Q(u, u) \geqslant 0$ is valid.

Lemma 4.5. Let $\Omega$ be a bounded set of finite perimeter in $M$ with $\Lambda=\Sigma \cup \Sigma_{0}$, where $\Sigma_{0}$ is a closed singular set such that $\mathcal{H}^{n-2}\left(\Sigma_{0}\right)=0$ or consists of isolated points, $\Sigma$ is a smooth, embedded hypersurface with boundary, and $\partial^{*} \Sigma=\partial \Sigma-\Sigma_{0}$ is contained in $\partial M$.

If $\Omega$ is stable and the cone $M$ is convex, then the index form defined in (4.1) satisfies $Q(u, u) \geqslant 0$ for any smooth bounded function $u$ on $\Sigma$ with mean zero and satisfying $|\nabla u|^{2},|\Delta u| \in L^{1}(\Sigma)$ and $\frac{\partial u}{\partial \nu} \in L^{1}\left(\partial^{*} \Sigma\right)$.

Proof. Let $u$ be any function under the hypotheses of the statement. By Lemma 4.4 we may integrate by parts to conclude that the index form $Q(u, u)$ in (4.1) equals $I(u, u)$ in (4.2). Hence, the proof finishes if we prove that $I(u, u) \geqslant 0$. To prove this inequality, we can proceed as in [MR, Lemma 3.3]. We use the sequence $\left\{\varphi_{\varepsilon}\right\}$ of Lemma 4.3 to construct a sequence of functions $\left\{u_{\varepsilon}\right\}_{\varepsilon>0}$ with mean zero and 
compact support in $\Sigma$, such that $\left\{u_{\varepsilon}\right\} \rightarrow u$ in the Sobolev space $H^{1}(\Sigma)$. By the stability of $\Omega$, we get $I\left(u_{\varepsilon}, u_{\varepsilon}\right) \geqslant 0$, which is equivalent to

$$
\int_{\Sigma}|\sigma|^{2} u_{\varepsilon}^{2} d \mathcal{H}^{n}+\int_{\partial^{*} \Sigma} \operatorname{II}(N, N) u_{\varepsilon}^{2} d \mathcal{H}^{n-1} \leqslant \int_{\Sigma}\left|\nabla u_{\varepsilon}\right|^{2} d \mathcal{H}^{n} .
$$

Note that $\operatorname{II}(N, N) \geqslant 0$ since $M$ is convex, and so we can take liminf in the inequality above and use Fatou's Lemma to deduce that

$$
\int_{\Sigma}|\sigma|^{2} u^{2} d \mathcal{H}^{n}+\int_{\partial^{*} \Sigma} \operatorname{II}(N, N) u^{2} d \mathcal{H}^{n-1} \leqslant \int_{\Sigma}|\nabla u|^{2} d \mathcal{H}^{n}<\infty,
$$

from which the proof follows.

Remark 4.6. Inequality $I(u, u) \geqslant 0$ for functions $u$ as in the lemma above was obtained by P. Sternberg and K. Zumbrun [SZ1] for stable regions with a singular set $\Sigma_{0}$ such that $\mathcal{H}^{n-2}\left(\Sigma_{0}\right)=0$. However, they did not treat the case of isolated singularities.

The following lemma will be useful in proving that the test function $u=1+$ $H\langle X, N\rangle$ satisfies the hypotheses of Lemma 4.5

Lemma 4.7. Let $\Omega$ be a bounded set of finite perimeter in $M$ with $\Lambda=\Sigma \cup \Sigma_{0}$, where $\Sigma_{0}$ is a closed singular set such that $\mathcal{H}^{n-2}\left(\Sigma_{0}\right)=0$ or consists of isolated points, $\Sigma$ is a smooth, embedded hypersurface with boundary, and $\partial^{*} \Sigma=\partial \Sigma-\Sigma_{0}$ is contained in $\partial M$.

Let $N$ be the normal along $\Sigma$ pointing into $\Omega,|\sigma|^{2}$ the squared sum of the principal curvatures associated to $N$ and II the second fundamental form of $\partial M-\{0\}$ with respect to the inner normal.

If $\Omega$ is stable and $M$ is convex, then $|\sigma|^{2} \in L^{1}(\Sigma)$ and $\operatorname{II}(N, N) \in L^{1}\left(\partial^{*} \Sigma\right)$.

Proof. We reason as in [MR, Lemma 3.3]. Clearly, it suffices to show that $\int_{W}|\sigma|^{2} d \mathcal{H}^{n}, \int_{W \cap \partial^{*} \Sigma} \operatorname{II}(N, N) d \mathcal{H}^{n-1}<\infty$, where $W$ is a small open neighborhood of $\Sigma_{0}$ in $\Sigma$. To prove this we take the function $f \equiv 1$ in $W$ and extend it on $\Sigma$ to a bounded function with mean zero and such that $|\nabla f|^{2} \in L^{1}(\Sigma)$. By applying Lemma 4.5 to $f$, we have

$$
\int_{W}|\sigma|^{2} d \mathcal{H}^{n}+\int_{W \cap \partial^{*} \Sigma} \operatorname{II}(N, N) d \mathcal{H}^{n-1} \leqslant \int_{\Sigma}|\nabla f|^{2} d \mathcal{H}^{n}<\infty .
$$

In order to compute the index form $Q(u, u)$ given by 4.1) for $u=1+H\langle X, N\rangle$, we need the following result

Lemma 4.8. Let $\Sigma$ be a smooth, embedded hypersurface with boundary in a cone $M$. Denote by $\partial^{*} \Sigma$ the set of points $x \in \partial \Sigma \cap(\partial M-\{0\})$ such that $\Sigma$ meets $\partial M$ orthogonally in a neighborhood of $x$. Let $N$ be a unit normal vector to $\Sigma, \nu$ the inner normal to $\partial^{*} \Sigma$ in $\Sigma, X(p)=p$ the position vector field on $\mathbb{R}^{n+1}$, and $g=\langle X, N\rangle$ the support function of $\Sigma$. Then we have

$$
\frac{\partial g}{\partial \nu}=-\mathrm{II}(N, N) g, \quad \text { in } \partial^{*} \Sigma
$$

where II is the second fundamental form of $\partial M-\{0\}$ with respect to the inner normal. 
Proof. Denote by $\nu^{*}$ the inward normal vector to $\partial M-\{0\}$. First note that $\left\langle X, \nu^{*}\right\rangle=0$ in $\partial M$. As $\Sigma$ meets $\partial M$ orthogonally in $\partial^{*} \Sigma$, we can differentiate the previous equality with respect to $N$. Denoting by $D$ the covariant derivative in $\mathbb{R}^{n+1}$ and taking into account that $D_{e} X=e$ for any vector $e$, we obtain

$$
\left\langle X, D_{N} \nu^{*}\right\rangle=0 \text { in } \partial^{*} \Sigma .
$$

Second, we differentiate the equality $\langle\nu, N\rangle=0$ with respect to a vector $v \in$ $T\left(\partial^{*} \Sigma\right)$, to get

$$
\mathrm{II}(N, v)+\mathrm{II}_{\Sigma}(\nu, v)=0,
$$

where $\mathrm{II}_{\Sigma}$ is the second fundamental form of $\Sigma$ with respect to $N$. We have used the fact that $\nu=\nu^{*}$ in $\partial^{*} \Sigma$. Taking into account the symmetry of II and $\mathrm{II}_{\Sigma}$, the equality above becomes

$$
\left\langle v, D_{N} \nu^{*}+D_{\nu} N\right\rangle=0, \quad \text { for any } \quad v \in T\left(\partial^{*} \Sigma\right),
$$

from which we conclude that

$$
D_{N} \nu^{*}+D_{\nu} N=a \nu-\operatorname{II}(N, N) N \quad \text { in } \quad \partial^{*} \Sigma .
$$

Finally, by using (4.6) and equality $\langle X, \nu\rangle=0$ in $\partial^{*} \Sigma$, we conclude that

$$
\frac{\partial g}{\partial \nu}=D_{\nu} g=\left\langle X, D_{\nu} N\right\rangle=\left\langle X, a \nu-\operatorname{II}(N, N) N-D_{N} \nu^{*}\right\rangle=-\mathrm{II}(N, N) g .
$$

Now we can prove the main result of this section

Theorem 4.9. Let $\Omega$ be a bounded set of finite perimeter in a convex cone $M$ with $\overline{\partial \Omega \cap M}=\Sigma \cup \Sigma_{0}$, where $\Sigma_{0}$ is a closed singular set such that $\mathcal{H}^{n-2}\left(\Sigma_{0}\right)=0$ or consists of isolated points, $\Sigma$ is a smooth, embedded hypersurface with boundary, and $\partial^{*} \Sigma=\partial \Sigma-\Sigma_{0}$ is contained in $\partial M$.

If $\Omega$ is stable, then either $\Omega$ is a round open ball in $M$, or the intersection of a ball centered at the vertex of the cone with $M$, or a half-ball in $M$ with boundary in a flat piece of $\partial M$.

Proof. Let $X(p)=p$ be the position vector field on $\mathbb{R}^{n+1}, N$ the normal to $\Sigma$ pointing into $\Omega$, and $g=\langle X, N\rangle$ the support function of $\Sigma$ with respect to the origin. Consider the smooth, bounded function $u$ on $\Sigma$, given by

$$
u=1+H g,
$$

where $H$ is the constant mean curvature of $\Sigma$ with respect to $N$.

Let us prove that $u$ satisfies the hypotheses of Lemma 4.5. First note that

$$
\nabla u=-H \sum_{i} k_{i}\left\langle X, e_{i}\right\rangle e_{i}, \quad \Delta u=|\sigma|^{2}-n H^{2}-|\sigma|^{2} u, \quad \frac{\partial u}{\partial \nu}=-\mathrm{II}(N, N) H g,
$$

where $e_{i}$ is a principal direction of $\Sigma$ with principal curvature $k_{i}$. The Laplacian $\Delta u$ has been computed from $[\overline{\mathrm{BdC}}$ Lemma 3.5]. The derivative $\partial u / \partial \nu$ is calculated by using Lemma4.8 By equation (4.7) and Lemma 4.7]we obtain $|\nabla u|^{2},|\Delta u| \in L^{1}(\Sigma)$ and $\partial u / \partial \nu \in L^{1}\left(\partial^{*} \Sigma\right)$. 
Second, note that $u=n^{-1} \operatorname{div} X^{T}$, where $X^{T}(p)$ denotes the tangent projection of $X(p)=p$ in $T_{p} \Sigma$. As $\Sigma$ is bounded, we can apply the Divergence Theorem in Lemma 4.4(i) to obtain

$$
\int_{\Sigma} u d \mathcal{H}^{n}=-n^{-1} \int_{\partial^{*} \Sigma}\langle X, \nu\rangle d \mathcal{H}^{n-1}=0
$$

since $X$ is tangent to $\partial M-\{0\}$ and $\nu$ coincides with the inner normal vector to $\partial M-\{0\}$. Formula (4.8) is known as the first Minkowski formula. This formula implies that $H \neq 0$ and enables us to show that $\Sigma$ is connected. Otherwise, let $\Sigma_{1}$ and $\Sigma_{2}$ be two components of $\Sigma$. Consider a locally constant nowhere vanishing function $v$ with mean zero on $\Sigma_{1} \cup \Sigma_{2}$. It is straightforward to check that we can insert the function $v$ in the index form $Q$ by Lemma 4.5 By the stability of $\Sigma$ we get $Q(v, v) \geqslant 0$. But

$$
Q(v, v)=\sum_{i=1,2}\left\{-\int_{\Sigma_{i}}|\sigma|^{2} c_{i}^{2} d \mathcal{H}^{n}-\int_{\partial^{*} \Sigma_{i}} \operatorname{II}(N, N) c_{i}^{2} d \mathcal{H}^{n-1}\right\}<0,
$$

for some constants $c_{1}, c_{2} \neq 0$, a contradiction.

As $u$ is a mean zero function and satisfies the hypotheses of Lemma 4.5 we can assert that $Q(u, u) \geqslant 0$. Now we compute $Q(u, u)$. By (4.7) we have $\Delta u+|\sigma|^{2} u=$ $|\sigma|^{2}-n H^{2}$, and so

$$
-\int_{\Sigma} u\left(\Delta u+|\sigma|^{2} u\right) d \mathcal{H}^{n}=-\int_{\Sigma}\left(|\sigma|^{2}-n H^{2}\right) d \mathcal{H}^{n}-H \int_{\Sigma}\left(|\sigma|^{2}-n H^{2}\right) g d \mathcal{H}^{n}
$$

In order to compute the last integral in the equality above we use Lemma 4.4(i) with the tangent vector field $Y=\nabla g$, and we get

$$
\int_{\Sigma}|\sigma|^{2} g d \mathcal{H}^{n}=-\int_{\Sigma} n H d \mathcal{H}^{n}-\int_{\partial^{*} \Sigma} \mathrm{II}(N, N) g d \mathcal{H}^{n-1},
$$

where we have employed Lemma 4.8 to compute $\partial g / \partial \nu$ and $[\overline{\mathrm{BdC}}$, Lemma 3.5] to compute $\Delta g$. We deduce, taking into account (4.8), that

$$
\int_{\Sigma}\left(|\sigma|^{2}-n H^{2}\right) g d \mathcal{H}^{n}=-\int_{\partial^{*} \Sigma} \mathrm{II}(N, N) g d \mathcal{H}^{n-1}
$$

which is known as the second Minkowski formula. On the other hand, the third equality in (4.7) gives us

$$
\frac{\partial u}{\partial \nu}+\operatorname{II}(N, N) u=\operatorname{II}(N, N) \quad \text { in } \quad \partial^{*} \Sigma
$$

Finally, by (4.9), (4.10) and (4.11) we conclude that

$$
0 \leqslant Q(u, u)=-\int_{\Sigma}\left(|\sigma|^{2}-n H^{2}\right) d \mathcal{H}^{n}-\int_{\partial^{*} \Sigma} \operatorname{II}(N, N) d \mathcal{H}^{n-1} .
$$

The last inequality implies, by the convexity of the cone and $[\mathrm{BdC}$, Lemma 3.2], that

$$
|\sigma|^{2} \equiv n H^{2} \text { and } \operatorname{II}(N, N) \equiv 0,
$$

and so $\Sigma$ is a connected piece of a totally umbilical sphere. Hence the normal vector $N$ to $\Sigma$ can be extended continuously to $\partial \Omega \cap M$ and, by [Gi, Theorem 4.11], we deduce that $\partial \Omega \cap M$ is a smooth hypersurface. From a connectedness argument we conclude that the singular set $\Sigma_{0}$ is empty and, therefore, $\Sigma$ is a compact connected piece of a round sphere. If $\partial \Sigma=\emptyset$, then $\Sigma$ is a sphere contained in $\bar{M}$. If $\partial \Sigma \neq \emptyset$ the conclusion follows from Lemma 4.10 below. 
Lemma 4.10. Let $M \subset \mathbb{R}^{n+1}$ be a convex cone and let $S \subset \bar{M}$ be a compact, connected subset of a sphere such that $\partial S \subset \partial M$ and the boundary of $S$ meets $\partial M$ orthogonally. Then, either $S$ is the intersection of a sphere centered at the vertex with the cone or a half-sphere centered at $\partial M$ and lying over a flat piece of $\partial M$.

Proof. Let $N$ be the inner normal to $S$. The linear subspace generated by a vector $e \in \mathbb{R}^{n+1}$ will be denoted by $L(e)$. For any $x \in S$, the normal line $x+L(N(x))$ to $S$ contains the center $x_{0}$ of $S$. Taking a point $y \in \partial S-\{0\}$ at maximum distance from the vertex of the cone and using the orthogonality condition, we deduce that $N(y)$ is proportional to $y$, and so $x_{0} \in \partial M \cup(-\partial M)$. Cutting with a plane passing through $0, y$ and containing $N(y)$ we see that the center of the sphere $x_{0} \in \partial M$.

If $x_{0}=0$, then $S$ is a sphere centered at the vertex intersected with $M$. If $x_{0} \neq 0$, then choose any $x \in \partial S-\{0\}$. The tangent hyperplane $\Pi_{x}$ to $\partial M$ at $x$ is a supporting hyperplane of the convex cone $M$ and contains the straight line $x+L(N(x))$ since $N(x)$ is tangent to $\partial M$ by the orthogonality condition. So $x_{0}, x \in \Pi_{x} \cap \partial M$ and we conclude that the segment line $\left[x_{0}, x\right]$ is contained in $\Pi_{x} \cap \partial M$ by the convexity of $M$. From here we get that $\partial S \subset \Pi_{0} \cap \partial M$, where $\Pi_{0}$ is the tangent hyperplane to $\partial M$ at $x_{0}$. Hence $\partial S$ is a great circle of $S$, and $\partial S$ bounds a flat region in $\Pi_{0} \cap \partial M$.

As a consequence of the classification of stable regions in a convex cone $M$, we can show which are the isoperimetric regions in $M$.

Theorem 4.11 ([LP]). Isoperimetric regions in a convex cone $M \subset \mathbb{R}^{n+1}$ different from a half-space are balls centered at the vertex intersected with the cone.

Proof. By Proposition 3.5 the existence of isoperimetric regions for any volume is guaranteed. Let $\Omega$ be an isoperimetric region. By Proposition 3.7 we know that $\Omega$ is a bounded set of finite perimeter. Moreover, by Proposition 2.4 the singular set $\Sigma_{0}$ satisfies $\mathcal{H}^{n-2}\left(\Sigma_{0}-\{0\}\right)=0$ and so $\mathcal{H}^{n-2}\left(\Sigma_{0}\right)=0$ if $n \geqslant 3$ or $\Sigma_{0} \subseteq\{0\}$ if $n=2$. As $\Omega$ is stable we conclude by Theorem 4.9 that $\Omega$ can be a ball, a half-ball lying over a flat piece of $\partial M$ or the intersection between a ball centered at the vertex and the cone. The proof finishes by using that the relative profile of the balls centered at the vertex intersected with the cone is better than the profile of the half-space.

An special case of a convex cone is a half-space of the Euclidean space.

Corollary 4.12. Let $\Omega$ be a bounded set of finite perimeter in a half-space $H \subset$ $\mathbb{R}^{n+1}$ such that $\overline{\partial \Omega \cap H}$ can be decomposed as a disjoint union $\Sigma \cup \Sigma_{0}$, where $\Sigma$ is a smooth, embedded hypersurface with boundary in the hyperplane $\partial H$, and $\Sigma_{0}$ is a closed set of singularities such that $\mathcal{H}^{n-2}\left(\Sigma_{0}\right)=0$ or consisting of isolated points.

If $\Omega$ is stable, then $\Omega$ is an open round ball contained in $H$ or a half-ball centered at $\partial H$.

The result above was obtained by P. Sternberg and K. Zumbrun SZ1] when the set $\Omega$ is a local minimizer of perimeter with a volume constraint. They did not use the fact that any local minimizer is stable. Their proof consists in reflecting the local minimizer with respect to $\partial H$ and use the classical isoperimetric inequality in $\mathbb{R}^{n+1}$. We do not know a previous proof of Corollary 4.12 in the literature. Of course the classification of stable regions in $H$ shows which are the isoperimetric regions in $H$. 
Corollary 4.13. Isoperimetric regions in a half-space $H \subset \mathbb{R}^{n+1}$ are the half-balls centered at the boundary of the half-space.

The arguments we have used in this section are also valid in the Euclidean space $\mathbb{R}^{n+1}$. They are even simpler since boundary terms do not appear, and allow us to prove

Corollary 4.14 ([SZ1]). Let $\Omega$ be a bounded set of finite perimeter in $\mathbb{R}^{n+1}$ such that $\partial \Omega=\Sigma \cup \Sigma_{0}$, where $\Sigma$ is a smooth, embedded hypersurface, and $\Sigma_{0}$ is a closed set of singularities such that $\mathcal{H}^{n-2}\left(\Sigma_{0}\right)=0$.

If $\Omega$ is stable, then $\Omega$ is a round ball in $\mathbb{R}^{n+1}$.

Remark 4.15. The result above was proved in $[\mathrm{BdC}]$ for smooth, compact, immersed hypersurfaces without singularities. P. Sternberg and K. Zumbrun proved in SZ1] that Corollary 4.14 holds.

Since the existence of isoperimetric regions in $\mathbb{R}^{n+1}$ can be obtained from general results [M3, Theorem 13.4], without appealing to the isoperimetric inequality in Euclidean space, we get

Corollary 4.16. Isoperimetric regions in $\mathbb{R}^{n+1}$ are round balls.

Problem. Consider the Clifford torus $T \subset \mathbb{S}^{3}$

$$
T=\left\{(x, y, z, t) \in \mathbb{R}^{4}: x^{2}+y^{2}=z^{2}+t^{2}=1 / 2\right\} .
$$

$\mathbb{S}^{3}-T$ is the union of two domains $C$ and $D$, which are isometric via the antipodal map and satisfy $\mathcal{H}^{3}(C)=\mathcal{H}^{3}(D)=\mathbf{c}_{3} / 2$, where $\mathbf{c}_{3}=\mathcal{H}^{3}\left(\mathbb{S}^{3}\right)$. Hence, if $M$ is the cone over $C$ we know by Proposition 3.5 that there exist isoperimetric regions in $M$ for any volume. We cannot apply our results since $M$ is nonconvex (in fact, at any point of $\partial M-\{0\}$ there are always two principal curvatures with opposite values).

Example. Let $M \subset \mathbb{R}^{n+1}$ be a cone over a smooth domain $C$ in $\mathbb{S}^{n}$ with $\mathcal{H}^{n}(C) \leqslant$ $\mathbf{c}_{n} / 2$. We know from Proposition 3.5 that isoperimetric regions exist in $M$ for any given volume. In [LP, Remark 1.3], an example of a nonconvex cone in which the balls centered at the vertex intersected with the cone do not minimize perimeter with a volume constraint is given.

\section{Appendix: An alternative proof of the characterization OF ISOPERIMETRIC REGIONS IN A CONVEX CONE}

Using the special form of the isoperimetric profile in a cone, we can give another proof of the characterization of isoperimetric regions in a convex cone.

Proof of Theorem 4.11. Let $\Omega$ be an isoperimetric region in the cone $M$ with volume $V_{0}>0$ (existence of $\Omega$ is guaranteed by Proposition 3.5). The set $\Omega$ is bounded by Proposition 3.7 and is stationary. Denote by $\Sigma$ the regular part of $\overline{\partial \Omega \cap M}$. Let $N$ be the normal along $\Sigma$ which points into $\Omega$, and let $H$ be the constant mean curvature of $\Sigma$ with respect to $N$. From statement (iii) in Proposition 2.4 we know that the singular set $\Sigma_{0}$ satisfies $\mathcal{H}^{n-2}\left(\Sigma_{0}\right)=0$ or $\Sigma_{0} \subseteq\{0\}$. Hence, we can consider the functions $\varphi_{\varepsilon}: \Sigma \rightarrow \mathbb{R}$ given by Lemma 4.3,

Fix $\varepsilon>0$ and take a vector field $X$ over the cone, such that $X(p) \in T_{p}(\partial M)$ whenever $p \in \partial M-\{0\}$ and $\left.X\right|_{\Sigma}=\varphi_{\varepsilon} N$. The flow of diffeomorphisms $\left\{f_{t}\right\}_{t}$ of $X$ 
induces a variation $\Omega_{t}=f_{t}(\Omega)$ of $\Omega$. Call $V(t)=\operatorname{vol}\left(\Omega_{t}\right)$ and $\mathcal{P}(t)=\mathcal{P}\left(\Omega_{t}\right)$. By the first variation formulae for perimeter and volume

$$
\mathcal{P}^{\prime}(t)=\left.\frac{d \mathcal{P}}{d t}\right|_{t}=-\int_{\Sigma_{t}} n H_{t}\left\langle X, N_{t}\right\rangle d \mathcal{H}^{n}-\int_{\Sigma_{t} \cap \partial M}\left\langle X, \nu_{t}\right\rangle d \mathcal{H}^{n-1}
$$

where $\nu_{t}$ is the inward normal vector to $\Sigma_{t} \cap \partial M$ in $\Sigma_{t}=f_{t}(\Sigma)$. We also obtain by (4.3)

$$
V^{\prime}(t)=\left.\frac{d V}{d t}\right|_{t}=\int_{\Omega_{t}} \operatorname{div} X d \mathcal{H}^{n+1}=-\int_{\Sigma_{t}}\left\langle X, N_{t}\right\rangle d \mathcal{H}^{n},
$$

so that $V^{\prime}(0)=-\int_{\Sigma} \varphi_{\varepsilon} d \mathcal{H}^{n}<0$. Hence we can write $t$ as a function of the volume $V=V(t)$ for $V$ close to $V_{0}$. Let $\mathcal{P}(V)=\mathcal{P}[t(V)]$. By (5.1) and (5.2) we have

$$
\left.\frac{d \mathcal{P}}{d V}\right|_{V=V_{0}}=\left(\int_{\Sigma} \varphi_{\varepsilon} d \mathcal{H}^{n}\right)^{-1}\left(\int_{\Sigma} n H \varphi_{\varepsilon} d \mathcal{H}^{n}\right)=n H .
$$

Note that, by the definition of the isoperimetric profile,

$$
\mathcal{P}^{(n+1) / n}(V) \geqslant I_{M}^{(n+1) / n}(V)
$$

is satisfied for $V$ close to $V_{0}$; moreover, as $\Omega$ is an isoperimetric region, both functions coincide in $V_{0}$. Thus

$$
\left.\frac{d \mathcal{P}^{(n+1) / n}}{d V}\right|_{V=V_{0}}>0,\left.\quad \frac{d^{2} \mathcal{P}^{(n+1) / n}}{d V^{2}}\right|_{V=V_{0}} \geqslant 0,
$$

where we have used that $I_{M}^{(n+1) / n}(V)$ is a linear function of $V$ (Proposition 3.1). By the first equality above and (5.3) we deduce that $H>0$.

Now, we shall compute $d^{2} \mathcal{P}^{(n+1) / n} / d V^{2}$ at $V=V_{0}$. A straightforward calculation gives us

$$
\left.\frac{d^{2} \mathcal{P}^{(n+1) / n}}{d V^{2}}\right|_{V=V_{0}}=\left(\frac{n+1}{n}\right) \mathcal{P}(\Omega)^{1 / n}\left\{\frac{1}{n} \mathcal{P}(\Omega)^{-1} \mathcal{P}^{\prime}\left(V_{0}\right)^{2}+\mathcal{P}^{\prime \prime}\left(V_{0}\right)\right\},
$$

so we only have to compute $\mathcal{P}^{\prime \prime}\left(V_{0}\right)$. This calculation requires second variation of perimeter and volume; a detailed development can be found in [SZ2 Theorem 2.5]. It is obtained

$$
\begin{aligned}
\mathcal{P}^{\prime \prime}\left(V_{0}\right)=\left.\frac{d^{2} \mathcal{P}}{d V^{2}}\right|_{V=V_{0}} & =\left(\int_{\Sigma} \varphi_{\varepsilon} d \mathcal{H}^{n}\right)^{-2} \\
& \times\left\{\int_{\Sigma}\left(\left|\nabla \varphi_{\varepsilon}\right|^{2}-|\sigma|^{2} \varphi_{\varepsilon}^{2}\right) d \mathcal{H}^{n}-\int_{\Sigma \cap \partial M} \operatorname{II}(N, N) \varphi_{\varepsilon}^{2} d \mathcal{H}^{n-1}\right\},
\end{aligned}
$$

where $|\sigma|^{2}$ is the squared sum of the principal curvatures associated to $N$, and II the second fundamental form of $\partial M-\{0\}$ with respect to the inner normal (recall that $N$ is tangent to $\partial M$ at the points in $\Sigma \cap \partial M$ since $\Sigma$ meets $\partial M$ orthogonally).

Taking into account (5.3), (5.6), (5.5), and the second inequality in (5.4), we get

$$
\begin{aligned}
\left(\frac{n+1}{n}\right) & \mathcal{P}(\Omega)^{1 / n} \times\left\{\mathcal{P}(\Omega)^{-2}\left(\int_{\Sigma} n H^{2} d \mathcal{H}^{n}\right)+\left(\int_{\Sigma} \varphi_{\varepsilon} d \mathcal{H}^{n}\right)^{-2}\right. \\
& \left.\times\left(\int_{\Sigma}\left(\left|\nabla \varphi_{\varepsilon}\right|^{2}-|\sigma|^{2} \varphi_{\varepsilon}^{2}\right) d \mathcal{H}^{n}-\int_{\Sigma \cap \partial M} \operatorname{II}(N, N) \varphi_{\varepsilon}^{2} d \mathcal{H}^{n-1}\right)\right\} \geqslant 0 .
\end{aligned}
$$


Now, passing the negative terms of the equation above to the right of the inequality and taking liminf when $\varepsilon \rightarrow 0$, we obtain, by Fatou's Lemma and Lemma 4.3,

$$
\int_{\Sigma}|\sigma|^{2} d \mathcal{H}^{n}+\int_{\Sigma \cap \partial M} \operatorname{II}(N, N) d \mathcal{H}^{n-1} \leqslant \int_{\Sigma} n H^{2} d \mathcal{H}^{n}<\infty
$$

from which we conclude that $\int_{\Sigma}|\sigma|^{2} d \mathcal{H}^{n}, \int_{\Sigma \cap \partial M} \operatorname{II}(N, N) d \mathcal{H}^{n-1}<\infty$ and

$$
-\int_{\Sigma}\left(|\sigma|^{2}-n H^{2}\right) d \mathcal{H}^{n}-\int_{\Sigma \cap \partial M} \operatorname{II}(N, N) d \mathcal{H}^{n-1} \geqslant 0 .
$$

Finally, from the convexity of the cone and [BdC Lemma 3.2], we obtain

$$
|\sigma|^{2} \equiv n H^{2}, \quad \operatorname{II}(N, N) \equiv 0
$$

and so $\Sigma$ is a union of pieces of totally umbilical spheres of the same radius $1 / H$. To prove that $\Sigma$ is compact and connected we can proceed as in Theorem 4.9 Finally, from Lemma 4.10 we conclude that $\Omega$ is either a ball contained in $M$, or a half-ball lying over a flat piece of $\partial M$, or a ball centered at the origin intersected with the cone. A simple comparison of the perimeters of the candidates finally shows that the last ones are the only isoperimetric regions.

Remark 5.1. The proof of Theorem 4.11 given in this section is also valid if the cone $M$ coincides with the Euclidean space $\mathbb{R}^{n+1}$. In this case all the boundary terms do not appear and the computations are even simpler. In this way we get another proof of the isoperimetric property of balls in Euclidean space.

\section{REFERENCES}

[A] F. J. Almgren, Jr., Existence and regularity almost everywhere of solutions to elliptic variational problems with constraints, Mem. Amer. Math. Soc. 4 (1976), no. 165, viii+199. MR 54:8420

[BdC] João Lucas Barbosa and Manfredo do Carmo, Stability of hypersurfaces with constant mean curvature, Math. Z. 185 (1984), no. 3, 339-353. MR 85k:58021c

[BM] Pierre Bérard and Daniel Meyer, Inégalités isopérimétriques et applications, Ann. Sci. École Norm. Sup. (4) 15 (1982), no. 3, 513-541. MR 84h:58147

[BZ] Yu. D. Burago and V. A. Zalgaller, Geometric inequalities, Grundlehren der Mathematischen Wissenschaften [Fundamental Principles of Mathematical Sciences], vol. 285, Springer-Verlag, Berlin, 1988, Translated from the Russian by A. B. Sosinskiü, Springer Series in Soviet Mathematics. MR 89b:52020

[Ch] Isaac Chavel, Eigenvalues in Riemannian geometry, Pure and Applied Mathematics, vol. 115, Academic Press Inc., Orlando, FL, 1984. MR 86g:58140

[Gi] Enrico Giusti, Minimal surfaces and functions of bounded variation, Birkhäuser Verlag, Basel, 1984. MR 87a:58041

[GMT] Eduardo Gonzalez, Umberto Massari, and Italo Tamanini, On the regularity of boundaries of sets minimizing perimeter with a volume constraint, Indiana Univ. Math. J. 32 (1983), no. 1, 25-37. MR 84d:49043

[Gr] Misha Gromov, Metric structures for Riemannian and non-Riemannian spaces, Progress in Mathematics, vol. 152, Birkhäuser Boston Inc., Boston, MA, 1999, based on the 1981 French original MR 85e:53051, with appendices by M. Katz, P. Pansu and S. Semmes, translated from the French by Sean Michael Bates. MR 2000d:53065

[G1] Michael Grüter, Boundary regularity for solutions of a partitioning problem, Arch. Rational Mech. Anal. 97 (1987), no. 3, 261-270. MR 87k:49050

[G2] - Optimal regularity for codimension one minimal surfaces with a free boundary, Manuscripta Math. 58 (1987), no. 3, 295-343. MR 88m:49032

[GJ] Michael Grüter and Jürgen Jost, Allard type regularity results for varifolds with free boundaries, Ann. Scuola Norm. Sup. Pisa Cl. Sci. (4) 13 (1986), no. 1, 129-169. MR 89d:49048 
[LP] Pierre-Louis Lions and Filomena Pacella, Isoperimetric inequalities for convex cones, Proc. Amer. Math. Soc. 109 (1990), no. 2, 477-485. MR 90i:52021

[M1] Frank Morgan, Regularity of isoperimetric hypersurfaces in Riemannian manifolds, Trans. Amer. Math. Soc. (to appear).

[M2] L Riemannian geometry, second ed., A K Peters Ltd., Wellesley, MA, 1998, A beginner's guide. MR 98i:53001

[M3] - Geometric measure theory, third ed., Academic Press Inc., San Diego, CA, 2000, A beginner's guide. MR 2001j:49001

[MJ] Frank Morgan and David L. Johnson, Some sharp isoperimetric theorems for Riemannian manifolds, Indiana Univ. Math. J. 49 (2000), no. 3, 1017-1041. MR 2002e:53043

[MR] Frank Morgan and Manuel Ritoré, Isoperimetric regions in cones, Trans. Amer. Math. Soc. 354 (2002), no. 6, 2327-2339 (electronic). MR 2003a:53089

[R1] Manuel Ritoré, Constant geodesic curvature curves and isoperimetric domains in rotationally symmetric surfaces, Comm. Anal. Geom. 9 (2001), no. 5, 1093-1138. MR 2003a:53018

[R2] - The isoperimetric problem in complete surfaces of nonnegative curvature, J. Geom. Anal. 11 (2001), no. 3, 509-517. MR 2002f:53109

[RV] Antonio Ros and Enaldo Vergasta, Stability for hypersurfaces of constant mean curvature with free boundary, Geom. Dedicata 56 (1995), no. 1, 19-33. MR 96h:53013

[R] César Rosales, Isoperimetric regions in rotationally symmetric convex bodies, Indiana U. Math. J. 52 (2003), no. 5, 1201-1214.

[S] Leon Simon, Lectures on geometric measure theory, Proceedings of the Centre for Mathematical Analysis, Australian National University, vol. 3, Australian National University Centre for Mathematical Analysis, Canberra, 1983. MR 87a:49001

[SZ1] Peter Sternberg and Kevin Zumbrun, A Poincaré inequality with applications to volumeconstrained area-minimizing surfaces, J. Reine Angew. Math. 503 (1998), 63-85. MR 99g:58028

[SZ2] - On the connectivity of boundaries of sets minimizing perimeter subject to a volume constraint, Comm. Anal. Geom. 7 (1999), no. 1, 199-220. MR 2000d:49062

[StZ] Edward Stredulinsky and William P. Ziemer, Area minimizing sets subject to a volume constraint in a convex set, J. Geom. Anal. 7 (1997), no. 4, 653-677. MR 99k:49089

[W] Henry C. Wente, A note on the stability theorem of J. L. Barbosa and M. Do Carmo for closed surfaces of constant mean curvature, Pacific J. Math. 147 (1991), no. 2, 375-379. MR 92g:53010

[Wh] Hassler Whitney, Geometric integration theory, Princeton University Press, Princeton, N. J., 1957. MR 19:309c

[Z] William P. Ziemer, Weakly differentiable functions, Graduate Texts in Mathematics, vol. 120, Springer-Verlag, New York, 1989, Sobolev spaces and functions of bounded variation. MR 91e:46046

Departamento de Geometría y Topología, Universidad de Granada, E-18071 Granada, SPAIN

E-mail address: ritore@ugr.es

Departamento de Geometría y Topología, Universidad de Granada, E-18071 Granada, SPAIN

E-mail address: crosales@ugr.es 